\title{
Professor Mario Stefanelli
}

Professor Mario Stefanelli passed away in the early hours of October 19, 2010, due to the complications of a hemorrhagic stroke.

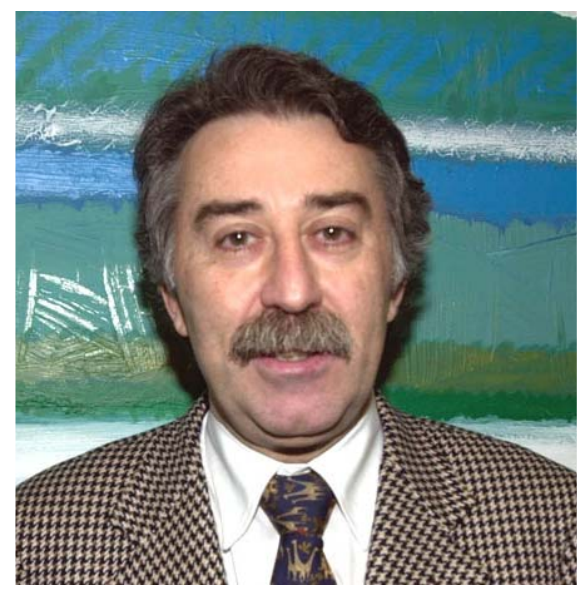

Even if he was seriously ill, the news took everyone by surprise, leaving his family, friends and collaborators dismayed and wordless. He was so lively, energetic and optimistic that it seemed impossible he would have gone away forever. Once before, six years ago, he had already won a similar disease thanks to his wonderful will power. So, his death seemed inconceivable. It is however impossible, for all of those who knew him, to forget his desire to talk, to discuss and to listen to anyone knocking on his door. He was a giant, both as a person and as a scientist.

Mario Stefanelli graduated cum laude in Electrical Engineering in 1969 at the University of Pavia, Italy. He soon became Full Professor of Automatic Control and later of Bioengineering, Medical Informatics and Artificial Intelligence in Medicine. His great passion for bioengineering made him a founding member of the Master's Degree and PhD Program in Bioengineering at the University of Pavia. Students have cherished him for his dedication and mentorship. In the same University, he had several and important official appointments: he directed the Department of Computer Engineering and Systems Science, where he established the Laboratory for Biomedical Informatics, which, thanks to his dedicated work, increased to the current size of forty people, including professors, $\mathrm{PhD}$ students and young research- ers; always close to quality issues, he was the prime mover of the University Evaluation Committee, that is now in charge of monitoring the educational and research activities of the entire University; from 2005 to 2009, he was Vice-Rector for the organizational models and the information systems; eventually, he promoted the creation of a joint degree course, collecting engineers, biologists and physicians, pursuing his belief on the multicultural nature of Bioengineering and Biomedical Informatics.

Prof. Stefanelli has been one of the most influential researchers in medical informatics.

He was one of the founders of the European Society for Artificial Intelligence in Medicine, which started its activity in Pavia in 1985 with its first European meeting.

He strictly collaborated with IMIA. After having been member of Medinfo SPCs, his influential role within IMIA has led him to be appointed as SPC chair of Medinfo 2004 together with Casimir Kulikowski. Medinfo 2004 is widely recognized as one the best IMIA conferences. He always fostered IMIA activities and worked with IMIA affiliated societies, including AMIA and EFMI. He has been the first Italian to be elected Fellow of the American College of Medical Informatics.

He coordinated several research projects funded by the European Commission and actively collaborated with many medical informatics laboratories in the world.

He has been a member of the editorial board of prestigious international journals, comprising the Journal of Biomedical Informatics, the International Journal of Medical Informatics, Artificial Intelligence in Medicine, and Methods of Information in Medicine.

A brief synthesis of his scientific career starts in the ' 70 s, when he gave important contributions to the mathematical modelling of erythropoiesis, using both quantitative methods and qualitative simulation methods, with applications to the diagnosis of anaemia. His specific interest to the problem of diagnostic reasoning motivated him to start a new research activity in the area of decision support systems and artificial intelligence in medicine. After a visit to the Casimir Kulikowski's labs at the
Rutgers University, Mario Stefanelli opened a Medical Informatics Labs at the University of Pavia, which soon became a leading laboratory worldwide First, the expert systems Anemia and Neoanemia were developed and tested, then the GAMES (General Architecture for Medical Expert Systems) European project gave rise to a new epistemological model of medical reasoning, including diagnosis, therapy planning and monitoring. The Medical Informatics Labs started to broaden their interests, including intelligent agents, probabilistic reasoning, temporal reasoning, telemedicine. In the last part of his scientific career, Prof. Stefanelli has devoted noteworthy efforts to the design of methods and tools able to support the entire process of patients' care. For this reason, he first studied the role of electronic medical records empowered by clinical guidelines, and second the impact of workflow systems in health care. In this context he coined the term "careflow" to denote all activities related to patient's care, which can be conveniently optimized by means of information-based solutions. He finally proposed to extend the careflow concept to manage continuity of care through the idea of "service flows".

He was a researcher, but he always promoted and supported technology transfer. His commitment to put research results into practice led to stable collaborations with several healthcare organisations, where the systems developed in his labs are currently implemented. During the last years he was working on the project of the Pavia technology park, which he thought as the crowning achievement of his career.

The entire medical informatics community sorely misses Prof. Stefanelli, a warm human being and a good friend to so many of us.

We enjoyed very good times working together, learning from his great vigour and intelligence.

Our thoughts, our memories and our prayers are for you, Mario.

\section{Acknowledgements}

We gratefully acknowledge Marco Tornielli for his help in revising the early versions of the paper.

Riccardo Bellazzi and Silvana Quaglini Dipartimento di Informatica e Sistemistica, Università di Pavia, Italy 